\title{
Enfrentamiento de la ingesta de cuerpo extraño en un Hospital Regional del sur de Chile
}

\author{
Albert Pastén G. ${ }^{1}$, Bárbara González F. ${ }^{1}$, \\ Pablo González J. ${ }^{1}$ y Óscar Figueroa R. ${ }^{1}$
}

\section{Management of foreign body ingestion at a Regional Hospital in southern Chile}

Introduction: Foreign body ingestion (FBI) is common in pediatrics and can lead to complications. The aim of the present study is to review the experience of our center in the management of these patients and to propose a treatment protocol. Material and Method: Retrospective, observational and descriptive cross-sectional study, in which the endoscopic reports and records of patients diagnosed with IFB managed by the Pediatric Endoscopy Unit of the Regional Clinical Hospital of Concepción between January 2013 and June 2017 (53 months) were reviewed, with a total of 40 cases. Data recording and analysis was performed with Microsoft ${ }^{\circledR}$ Excel $2016^{\circledR}$ for MacOS ${ }^{\circledR}$. Results: The average age was 3,96 \pm 3,24 years. The statistical fashion was 2 years. All patients underwent a upper gastrointestinal endoscopy. The most frequent FB was the coin $(61,9 \%)$. In 6 opportunities $(14,2 \%)$ was a button batery. FB retention was in the proximal third of the esophagus $(30,9 \%)$, the gastric body $(26,2 \%)$ and the distal third of the esophagus $(14,2 \%)$, being extracted mainly with foreign body clamp (90,4\%). The 57,5\% showed some injury related to the location of the FB, being the most serious the produced by button batery. Conclusion: FBI in children is a potentially dangerous entity, so it becomes necessary to have a pediatric endoscopy unit and treatment protocols to avoid complications, especially with high-risk objects.

Key words: foreign body ingestion; pediatric endoscopy; button cell; protocol; treatment.

\section{Resumen}

Introducción: La ingesta de cuerpos extraños (ICE) es frecuente en pediatría y puede llevar a complicaciones. Nuestro objetivo es revisar la experiencia de nuestro centro en el manejo de estos pacientes y proponer un protocolo de tratamiento. Material y Método: Estudio transversal retrospectivo, observacional y descriptivo, en el cual se revisaron los informes endoscópicos y fichas de pacientes con diagnóstico de ICE manejados por la Unidad de Endoscopia Pediátrica del Hospital Clínico Regional de Concepción entre enero de 2013 y junio de 2017 (53 meses), totalizando 40 casos. El registro y análisis de los datos se realizó con Microsoft ${ }^{\circledR}$ Excel $2016^{\circledR}$ para MacOS ${ }^{\circledR}$. Resultados: El promedio de edad fue de 3,96 \pm 3,24 años. La moda estadística fue de 2 años. A todos los pacientes se les realizó una endoscopia digestiva alta. El CE más frecuente fue la moneda $(61,9 \%)$. En 6 casos $(14,2 \%)$ fue una pila de botón. La retención de CE fue en tercio proximal del esófago (30,9\%), el cuerpo gástrico $(26,2 \%)$ y el tercio distal del esófago $(14,2 \%)$, extrayéndose principalmente con pinza de cuerpo extraño (90,4\%). El 57,5\% evidenció alguna lesión relacionada a la ubicación del CE, siendo la más grave la producida por pila de botón. Conclusión: La ICE en niños es una entidad potencialmente peligrosa, por lo que se hace necesario disponer de una unidad de endoscopia pediátrica y protocolos de manejo para evitar complicaciones, sobre todo con objetos de alto riesgo.

Palabras clave: ingesta cuerpo extraño; endoscopia pediátrica; pila de botón; protocolo; tratamiento.
'Hospital Clínico Regional de Concepción.

Recibido el 28 de noviembre de 2017, aceptado para publicación el 17 de abril de 2018.

Correspondencia a: Albert Pastén González apasteng@udec.cl 


\section{Introducción}

La curiosidad es parte del desarrollo psicomotor de los niños, lo que los lleva a explorar el mundo que los rodea sin la concepción del peligro. Distintos factores, tanto conductuales como de su anatomía y fisiología orales, hacen que el riesgo de ingesta de cuerpos extraños (ICE) sea mayor en este grupo etario ${ }^{1}$.

Este problema de salud es de gran importancia, pues según el reporte anual 2015 de la American Association of Poison Control, se registraron 81.825 casos de ICE durante ese año en EE. UU. en menores de 20 años, siendo 68.371 de éstos en menores de 5 años ${ }^{2}$.

Las monedas son el cuerpo extraño (CE) más frecuentemente ingerido por los niños, y los CE más peligrosos son las pilas de botón, los objetos con punta y los trozos de hueso ${ }^{3}$, que pueden generar morbilidad y mortalidad asociada.

El Hospital Clínico Regional de Concepción (HCRC), ubicado en la Región del Bío Bío, Chile, es un centro terciario de alta complejidad, a cargo de resolver las patologías más complejas de una población que bordea los 2 millones de habitantes, por lo que requiere ofrecer los más altos estándares de cuidado para sus usuarios. Es así como en 2013 se inició la implementación de la primera Unidad de Endoscopia Pediátrica (UEP) del sur de Chile, en el Servicio de Cirugía Infantil y Ortopedia de este centro, convirtiéndose en la referencia regional para el manejo de los pacientes con ICE.

El objetivo de nuestro trabajo es revisar la experiencia acumulada en estos 4 años y 5 meses de manejo de pacientes pediátricos con ICE, planteando los protocolos de enfrentamiento de este problema según CE ingerido, adaptando las recomendaciones internacionales a nuestra realidad local.

\section{Material y Métodos}

Este estudio tiene un diseño de tipo retrospectivo, observacional y descriptivo. Se revisaron los informes endoscópicos de los pacientes con diagnóstico de ICE recibidos y manejados por la UEP del HCRC entre enero de 2013 y junio de 2017 (53 meses), desde donde se obtuvieron los datos para localizar sus fichas clínicas electrónicas.

Se incluyó en el estudio a 40 pacientes, de entre 8 meses y 12 años, que corresponden al total de pacientes con ICE tratados por nuestra UEP en el periodo anteriormente señalado. No se definieron criterios de exclusión del estudio.
Se registraron variables demográficas (sexo, edad), antecedentes de la ICE (fecha de ocurrencia, tipo y número de $\mathrm{CE}$ ingeridos) y antecedentes endoscópicos (ubicación del CE, lesiones asociadas, pinza utilizada para extracción, tiempo del procedimiento, complicaciones inmediatas).

A todos los pacientes se les practicó una EDA flexible por uno de los dos cirujanos pediátricos de la UEP, en quirófano, bajo anestesia general. Éstos reciben a los pacientes durante sus guardias en la Unidad de Emergencia Pediátrica del HGGB, y funcionan en modalidad de llamado cuando acuden pacientes fuera de sus correspondientes guardias.

El instrumental disponible en la UEP para extracción de CE son pinzas de cuerpo extraño tipo Raptor $^{\circledR}$, cestas endoscópicas tipo Rothnet ${ }^{\circledR}$ y asas endoscópicas.

El registro de datos y el análisis estadístico de los mismos se realizó en la aplicación Microsoft ${ }^{\mathrm{B}}$ Excel $2016^{\circledR}$ para $\mathrm{MacOS}^{\circledR}$ (Redmond, Washington, EE. UU.). Se calcularon la media de edad y su DE, la frecuencia por sexo, y la media de tiempo del procedimiento. Datos como el tipo y número de $\mathrm{CE}$ ingeridos, la ubicación del CE y las lesiones asociadas se ordenaron en tablas expositivas.

\section{Resultados}

Se extrajeron $42 \mathrm{CE}$ de los 40 pacientes estudiados. 19 de éstos fueron niñas $(47,5 \%)$ y 21 fueron niños (52,5\%). El promedio de edad fue de 3,96 $\pm 3,24$ años (rango 8 meses - 12 años). La moda estadística fue de 2 años. Ninguno de los pacientes presentaba comorbilidades de interés.

En la Tabla 1 se muestran los CE más frecuentemente encontrados en las EDA realizadas. Veinticuatro de los pacientes ingirieron 26 monedas $(61,9 \%)$, en diferente cuantía y tamaño; la moneda de 10 pesos fue la más ingerida, y tiene un diámetro de $21 \mathrm{~mm}$. En 6 oportunidades (14,2\%) el CE ingerido fue una pila de botón. La Figura 1 ilustra algunos de los CE encontrados.

En la Tabla 2 se muestran las ubicaciones donde fueron hallados los CE extraídos, las edades promedio en cada una de ellas, y el tipo de CE ingerido. Los sitios más frecuentes fueron el estómago $(42,8 \%)$ y el tercio proximal del esófago $(28,5 \%)$. El $\mathrm{CE}$ se encontró en el tercio medio del esófago y en el estómago de los niños más pequeños $(2,22 \pm 1,68$ y $2,37 \pm 1,66$ años, respectivamente). En los niños más grandes, se impactó en el tercio distal esofágico $(8,33 \pm 4,08$ años $)$. 
Tabla 1. Tipos de cuerpo extraño ingeridos

\begin{tabular}{|lc|}
\hline Tipo & Número \\
\hline Moneda & 26 \\
\hline Pila botón & 6 \\
\hline Antena metálica & 1 \\
\hline Aro & 1 \\
\hline Argolla metálica & 1 \\
\hline Hueso de conejo & 1 \\
\hline Llave de cerradura & 1 \\
\hline Cortauñas & 1 \\
\hline Alfiler de gancho & 1 \\
\hline Clavo & 1 \\
\hline Pila AAA & 1 \\
\hline Medalla & 1 \\
\hline
\end{tabular}

La totalidad de los CE ingeridos fueron extraídos en el primer procedimiento endoscópico. En 38 de los 42 CE ingeridos $(90,4 \%)$ fueron extraídos con pinza de cuerpo extraño, 3 de ellos $(7,2 \%)$ se extrajeron con asa endoscópica, y $1(2,4 \%)$ se extrajo con cesta endoscópica. El tiempo promedio de demora del procedimiento fue de 15,35 $\pm 10,6 \mathrm{~min}$ (rango 5-50 $\mathrm{min})$.

En 23 pacientes $(57,5 \%)$ se evidenció alguna lesión relacionada a la ubicación del CE encontrado. Las lesiones más graves fueron quemaduras producidas por pilas de botón, en 3 pacientes. El espectro de lesiones que se hallaron en los estudios endoscópicos se representa en la Figura 2, y algunos hallazgos endoscópicos se muestran en la Figura 3.

\section{Discusión}

Demográficamente, los datos obtenidos en nuestro estudio son similares a los reportados en la literatura ${ }^{4,5}$, con un promedio de edad en torno a los 4 años, un discreto predominio masculino entre los pacientes, y un peak de incidencia en torno a los 2 años de vida.

La gran mayoría de los CE ingeridos son monedas, lo que también concuerda con los datos publicados a nivel internacional ${ }^{4,5}$. Durante el período de estudio 6 de nuestros pacientes ingresaron por ingesta de pila de botón, lo que supone quizá el mayor desafío al que puede enfrentarse un endos-

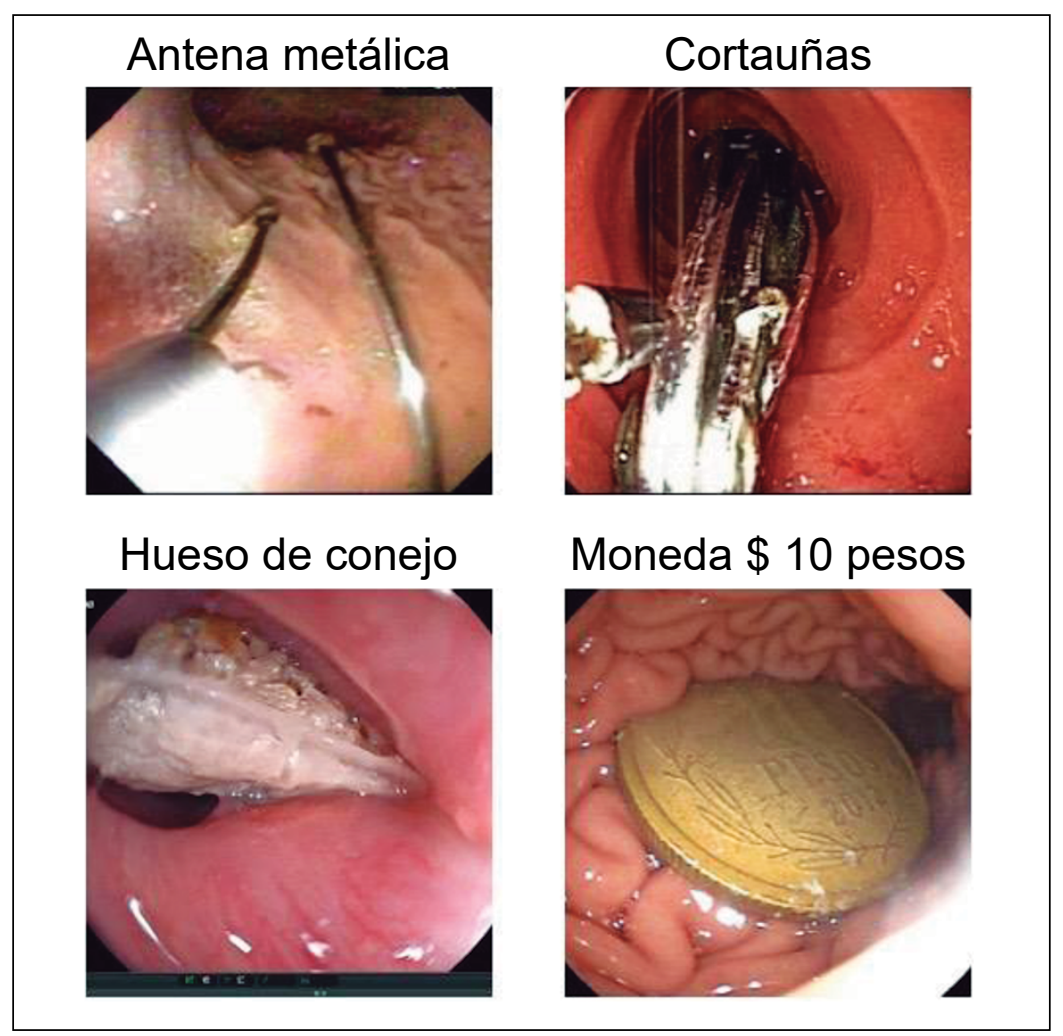

Figura 1. Imágenes endoscópicas de algunos de los CE extraídos.

Tabla 2. Ubicación de los cuerpos extraños ingeridos según edad y tipo

\begin{tabular}{|c|c|c|c|c|}
\hline Ubicación & Edad promedio $\pm \mathbf{D E}$ & CE & n & Total \\
\hline TPE & $4,36 \pm 3,23$ & $\begin{array}{l}\text { Moneda } 10 \\
\text { Moneda } 50 \\
\text { Moneda } 100 \\
\text { Pila botón } \\
\text { Otros }\end{array}$ & $\begin{array}{l}8 \\
1 \\
1 \\
1 \\
1\end{array}$ & 12 \\
\hline TME & $2,22 \pm 1,68$ & $\begin{array}{l}\text { Moneda } 100 \\
\text { Pila botón } \\
\text { Otros }\end{array}$ & $\begin{array}{l}1 \\
1 \\
1\end{array}$ & 3 \\
\hline TDE & $8,33 \pm 4,08$ & $\begin{array}{l}\text { Moneda } 10 \\
\text { Moneda } 50 \\
\text { Moneda } 100 \\
\text { Pila botón } \\
\text { Otros }\end{array}$ & $\begin{array}{l}2 \\
1 \\
1 \\
1 \\
1\end{array}$ & 6 \\
\hline Estómago & $2,37 \pm 1,66$ & $\begin{array}{l}\text { Moneda } 5 \\
\text { Moneda } 10 \\
\text { Moneda } 100 \\
\text { Pila botón } \\
\text { Otros }\end{array}$ & $\begin{array}{l}8 \\
2\end{array}$ & 18 \\
\hline Duodeno & $4 \pm 1,73$ & Otros & 3 & 3 \\
\hline Total & & & & 42 \\
\hline
\end{tabular}

TPE: tercio proximal del esófago; TME: tercio medio del esófago; TDE: tercio distal del esófago; DE: desviación estándar; CE: cuerpo extraño. 


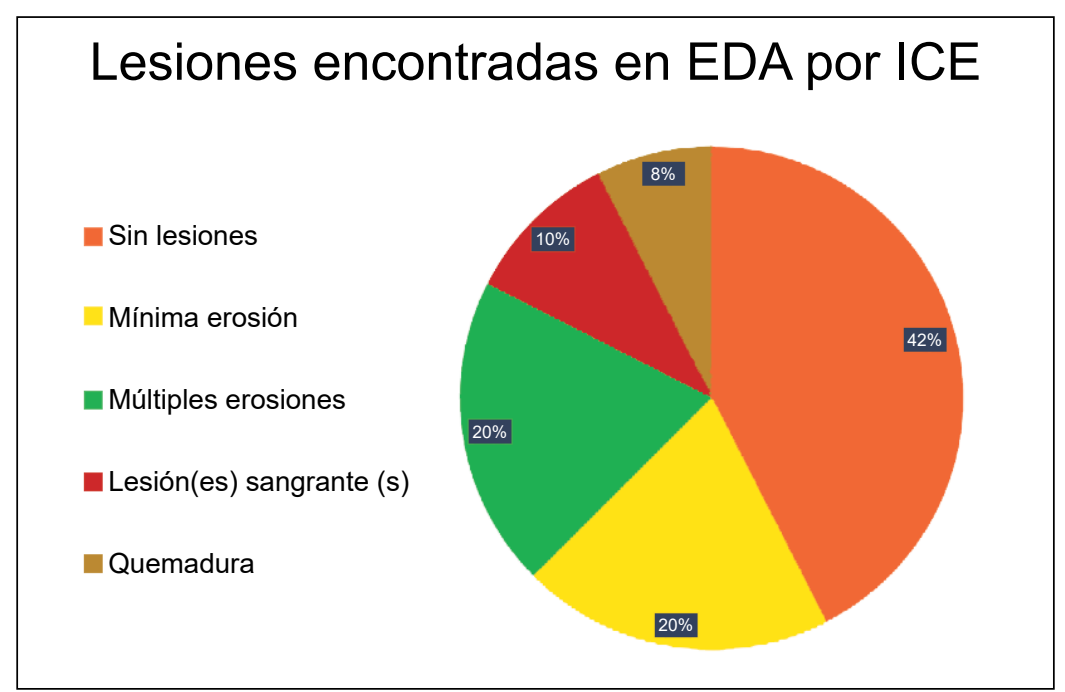

Figura 2. Representación gráfica de las lesiones encontradas en pacientes con ICE.

\section{Quemadura esofágica}

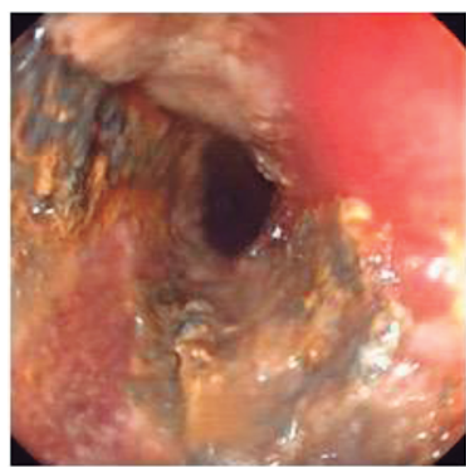

Erosión sangrante

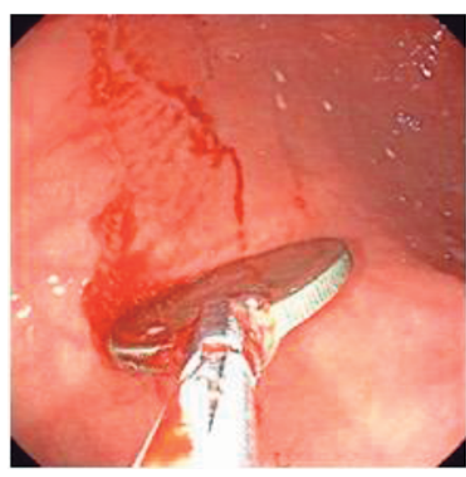

Erosión duodenal

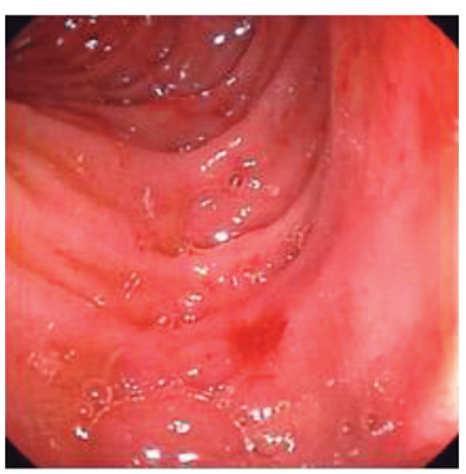

Figura 3. Imágenes de procedimientos endoscópicos con lesiones atribuibles a ICE. copista pediátrico. Varios autores han propuesto algoritmos de manejo para este tipo de pacientes, con el fin de minimizar la morbilidad y mortalidad asociada $^{6,7}$.

Varios estudios mencionan el sitio donde se localizó endoscópicamente el $\mathrm{CE}$ ingerido ${ }^{4,8,9}$. El esófago es el sitio donde los CE se encontraron con mayor frecuencia (50\%) siendo el tercio superior el que concentró los casos. Destaca en nuestra serie la cantidad de CE hallados en el estómago (42,8\%), lo que no concuerda con la literatura al respecto. El tamaño de los objetos ingeridos fue diverso en todos los grupos, si ordenamos a los pacientes respecto del sitio donde fue encontrado el CE, no impresionando haber una relación entre la edad, el lugar de impactación y el tamaño del objeto en nuestra serie.

Durante el tiempo estudiado, no se requirió repetir un procedimiento para extraer un $\mathrm{CE}$ ingerido. Los instrumentos endoscópicos de los que disponemos fueron suficientes a la hora de practicar cada extracción, siendo similares a los que se han descrito en otros estudios ${ }^{10}$.

El tiempo que tomó realizar cada procedimiento no fue registrado en la bibliografía revisada, por lo que nuestros datos no podrían ser comparados.

La mayoría de los pacientes de nuestra serie presentaron alguna lesión que se podría correlacionar con el CE ingerido, pues éste se encontraba en la vecindad de la disrupción mucosa. El mecanismo de lesión de CE punzantes puede ser el daño directo o el decúbito, siendo este último el determinante de disrupción mucosa por objetos romos, como las monedas. Por otro lado, las pilas de botón podrían lesionar los tejidos por transmisión eléctrica, depósito de sus electrolitos, y daño por decúbito $^{6}$. En nuestros pacientes, las lesiones más graves se observaron en los que ingirieron pilas de botón, presentándose con quemaduras circunferenciales sin perforación, que requirieron de ingreso en la Unidad de Paciente Crítico. El resto de las lesiones encontradas se pueden asociar a decúbito del CE extraído, pues ninguno de los pacientes de nuestra serie tenía alguna patología que pudiera explicar dichas lesiones.

\section{Protocolo}

Las conductas que deben seguirse en caso de ICE están bien establecidas, en condiciones ideales de recurso técnico y humano ${ }^{1,6,9-12}$. Nuestra UEP inició su implementación hace 4 años con el entrenamiento de dos cirujanos en endoscopia diagnóstica y terapéutica, siguiendo con la compra de los equipos necesarios. Considerando lo anterior, hemos tenido que adaptar las conductas recomendadas a nuestra 

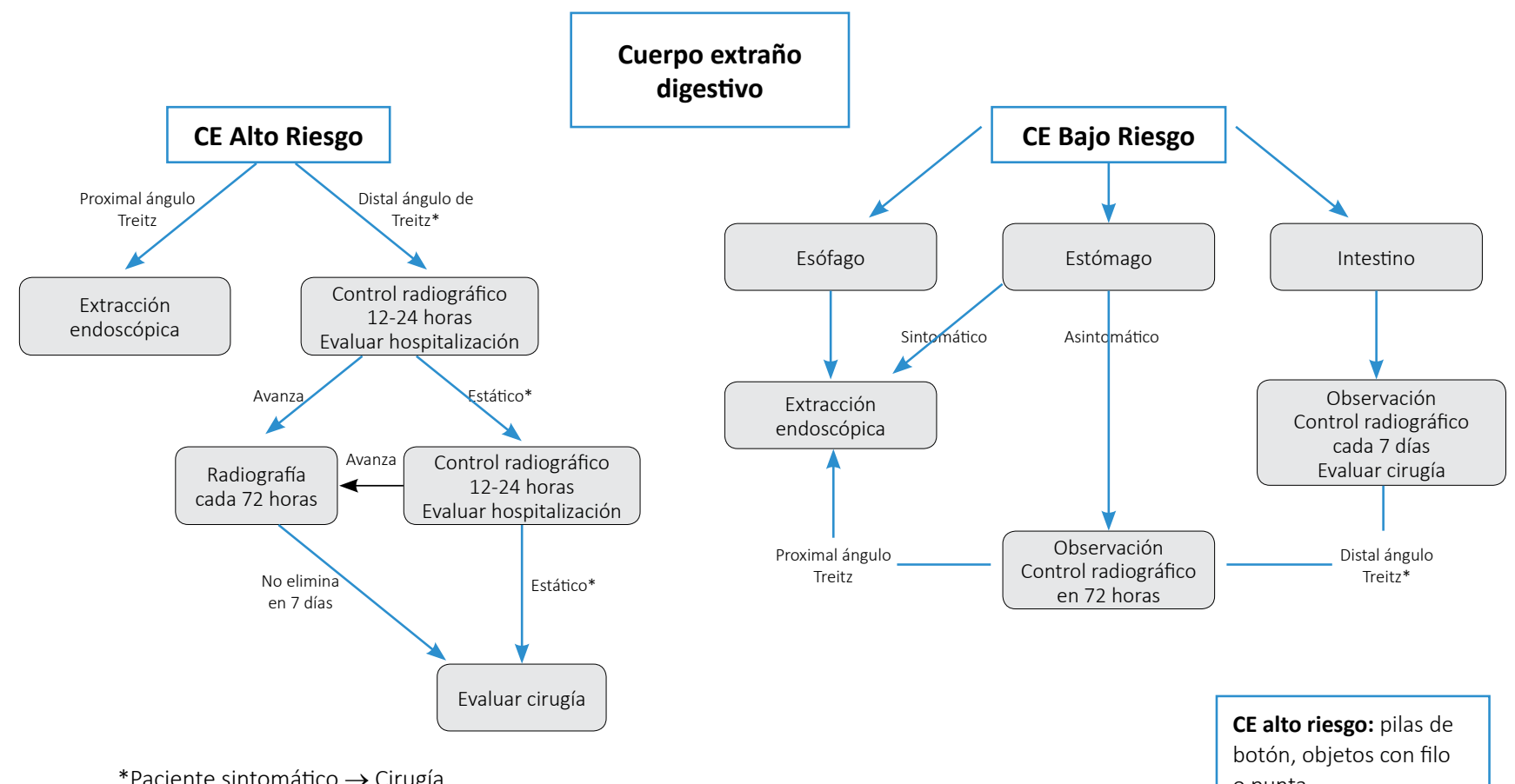

*Paciente sintomático $\rightarrow$ Cirugía

CE alto riesgo: pilas de botón, objetos con filo o punta

Figura 4. Algoritmo de actuación ante una ICE en nuestro centro.

realidad, que dista de las condiciones disponibles en centros de mayores recursos.

En la Figura 4 se detallan las conductas que se toman respecto de la sospecha de ICE, dependiendo del tipo y peligrosidad del CE, la sintomatología y el sitio donde se aloja al momento del diagnóstico.

Una vez que el motivo de consulta y la anamnesis sugiere ICE, se solicita una radiografía cervical, de tórax y abdomen frontal y lateral. Es importante que la placa incluya estas tres regiones, para poder apreciar el CE en todo su trayecto. Una imagen de $\mathrm{CE}$ que se ve de lado en proyección frontal sugiere $\mathrm{CE}$ en vía aérea, por lo que se solicita evaluación por otorrinolaringólogo.

Una vez confirmado el CE radiopaco en la vía digestiva, se debe determinar su riesgo de perforar la mucosa; cuando el riesgo es alto, la conducta se define según su ubicación. Si se encuentra proximal al ángulo de Treitz, está indicada la extracción endoscópica inmediata. Si los endoscopistas pediátricos entrenados no están de guardia, son llamados para realizar la extracción.

Cuando el CE está distal al ángulo de Treitz, está indicado el ingreso para observación radiográfica en 6 horas; si el CE avanza, es seguro dar el alta con control en el Servicio de Urgencias hasta que el CE sea expulsado por vía rectal. Si no avanza o aparecen signos de irritación peritoneal, se debe explorar quirúrgicamente, ya sea en forma abierta o videoasistida.

En caso de que el CE sea de bajo riesgo, la decisión de extraer se tomará según su ubicación. Si está en esófago, o está en estómago y es sintomático, se ingresa y se programa la extracción endoscópica dentro de las siguientes 24 horas. Si el CE gástrico es asintomático, se controla con radiografía en 2-3 días y se programa extracción en caso de encontrarse proximal al ángulo de Treitz.

En caso de CE en intestino o CE hallado distal al ángulo de Treitz en control radiográfico de CE gástrico, se indica control ambulatorio con observación de deposiciones. En caso de no encontrar el CE en 7 días, está indicado un nuevo control radiográfico. $\mathrm{Si}$ en dos controles seriados el CE permanece en el mismo lugar, se deberá considerar la exploración quirúrgica con ayuda de intensificador de imagen, según cada caso. 


\section{Conclusiones}

La ICE es una entidad clínica que se observa con cierta frecuencia en nuestro medio, dado el acceso de los niños a elementos pequeños, y a la poca conciencia que tienen los adultos ante el peligro que conllevan.

Disponer de instrumental endoscópico pediátrico y neonatal, además de personal altamente especializado en su utilización, nos convierte en el centro de referencia regional para el manejo de estos pacientes, evitando así las graves consecuencias que podría acarrear la ingesta de un CE con alto potencial de perforación.

Siendo el objetivo de nuestra UEP el recuperar y mantener la salud de los niños y adolescentes afectados de patologías que se puedan manejar en forma endoscópica, hace que la capacidad de manejar la
ICE infantil se convierta en uno de los propósitos fundamentales de nuestro desempeño diario.

\section{Responsabilidades éticas}

Protección de personas y animales. Los autores declaran que para esta investigación no se han realizado experimentos en seres humanos ni en animales.

Confidencialidad de los datos. Los autores declaran que en este artículo no aparecen datos de pacientes.

Derecho a la privacidad y consentimiento informado. Los autores declaran que en este artículo no aparecen datos de pacientes.

Conflictos de interés: Sin conflicto de interés.

Financiamiento: Sin financiamiento.

\section{Referencias}

1. Green S. Ingested and Aspirated Foreign Bodies. Pediatr Rev 2015;36:430-6.

2. Mowry J, Spyker D, Brooks D, Zimmerman A, Schauben J. 2015 Annual Report of the American Association of Poison Control Centers' National Poison Data System (NPDS): 33rd Annual Report. ClinToxicol. 2016;54:924-1109.

3. Peters N, Mahajan J, Bawa M, Chabbra A, Garg R, Rao K. Esophageal perforations due to foreign body impaction in children. J Pediatr Surg. 2015;50:1260-3.

4. Denney W, Ahmad N, Dillard B, Nowicki M. Children Will Eat the Strangest Things. Pediatr Emerg Care. 2012;28:731-4.

5. Kennedy R, Starker R, Feldman K,
Tashiro J, Pérez E, Mendoza F, et al. Cost varies with procedure type in pediatric GI foreign bodies. J Pediatr Surg. 201;52:410-13.

6. Martínez-Criado Y, Millán López A, Valladares J, De Agustín J. Impactación esofágica de pilas de botón en la infancia. ¡Cómo evitar la tragedia! Cir Pediatr 2013;26:142-5.

7. Leinwand K, Brumbaugh D, Kramer R. Button Battery Ingestion in Children: A Paradigm for Management of Severe Pediatric Foreign Body Ingestions. Gastrointest Endosc Clin N Am. 2016;26:99-118.

8. Sink J, Kitsko D, Mehta D, Georg M, Simons J. Diagnosis of Pediatric Foreign Body Ingestion: Clinical Presentation, Physical Examination, and Radiologic
Findings. Ann Otol Rhinol Laryngol. 2016;125:342-50.

9. Russell R, Lucas A, Johnson J, Yannam G, Griffin R, Beierle E, et al. Extraction of esophageal foreign bodies in children: rigid versus flexible endoscopy. Pediatr Surg Int. 2014;30:41722.

10. Muñoz M, Maluje R, Saitua F. Cuerpo extraño gastrointestinal en niños. Rev Chil Pediatr 2014;85:682-9.

11. Berdan E, Sato T. Pediatric Airway and Esophageal Foreign Bodies. Surg Clin N Am 2017;97:85-91.

12. Gershman G, Thomson M, Ament M. Therapeutic upper GI endoscopy. En: Practical Pediatric Gastrointestinal Endoscopy. 2012; $2^{\text {nd }}$ ed. Oxford: WileyBlackwell. Pp.82-103. 